REGARDS

SUR LECONOMIE ALLEMANDE

BULLETIN ECONOMIQUE DUCIRAC

\section{Regards sur l'économie allemande}

Bulletin économique du CIRAC

$96 \mid 2010$

Varia

\title{
L’Allemagne sort de la récession
}

Isabelle Bourgeois

\section{(2) OpenEdition}

Journals

Édition électronique

URL : http://journals.openedition.org/rea/4076

DOI : $10.4000 /$ rea. 4076

ISBN : 978-2-8218-0888-1

ISSN : 1965-0787

Éditeur

CIRAC

Édition imprimée

Date de publication : 1 mai 2010

Pagination : 3-4

ISSN : 1156-8992

Référence électronique

Isabelle Bourgeois, «L'Allemagne sort de la récession», Regards sur l'économie allemande [En ligne], 96 | mai 2010, mis en ligne le 26 mai 2010, consulté le 15 septembre 2020. URL : http://

journals.openedition.org/rea/4076 


\section{L'Allemagne sort de la récession}

"La reprise se poursuit - les risques restent grands ». C'est sous ce titre que les Instituts ont présenté le 15 avril leur rapport de printemps. Après une chute spectaculaire au plus fort de la crise mondiale, les exportations ont redémarré, tirées par l'expansion des économies émergentes. La demande intérieure frémit: les investissements reprennent et la consommation des ménages reste stable. Quant au marché du travail, il est «étonnamment robuste ", soulignent les Instituts. L'économie allemande devrait donc voir son PIB croître de $1,5 \%$ cette année, puis de 1,4\%. "L'économie allemande a renoué avec la croissance, et nous observons un petit miracle sur le marché de l'emploi », affirmait de son côté le ministre fédéral de l'Economie en présentant le rapport du gouvernement fédéral qui envisage, lui, des taux de respectivement $1,4 \%$ et $1,6 \%$. La Commission européenne prévoit $1,2 \%$ et $1,6 \%$ pour la $\operatorname{RFA}(0,9 \%$ et $1,5 \%$ pour la zone euro).

\begin{tabular}{|c|c|c|c|c|}
\hline \multicolumn{5}{|c|}{ Prévisions du rapport de printemps 2010 du groupe de travail des Instituts économiques } \\
\hline & 2008 & 2009 & 2010 & 2011 \\
\hline \multicolumn{5}{|c|}{ (variation en \% par rapport à l'année précédente) } \\
\hline $\begin{array}{l}\text { PIB } \\
\text { Ouest (Berlin inclus) } \\
\text { Est } \\
\text { Consommation privée } \\
\text { Consommation publique } \\
\text { Investisssements bruts } \\
\quad \text { biens d'équipement } \\
\quad \text { construction } \\
\quad \text { autres } \\
\text { Demande intérieure } \\
\text { Exportations } \\
\text { Importations } \\
\text { Prix à la consommation }(2000=\text { indice } 100) \\
\text { Coûts salariaux unitaires }\end{array}$ & $\begin{array}{l}1,3 \\
1,3 \\
1,0 \\
0,4 \\
2,1 \\
3,1 \\
3,3 \\
2,6 \\
5,3 \\
1,7 \\
2,9 \\
4,3 \\
2,6 \\
2,2\end{array}$ & $\begin{array}{r}-5,0 \\
-5,2 \\
-3,5 \\
0,2 \\
3,0 \\
-8,9 \\
-20,5 \\
-0,8 \\
4,9 \\
-2,1 \\
-14,2 \\
-8,9 \\
0,4 \\
5,2\end{array}$ & $\begin{array}{r}1,5 \\
1,5 \\
1,1 \\
-0,4 \\
1,6 \\
1,4 \\
2,2 \\
0,4 \\
6,2 \\
0,6 \\
7,1 \\
5,5 \\
0,9 \\
-0,6\end{array}$ & $\begin{array}{r}1,4 \\
1,4 \\
1,3 \\
0,8 \\
1,1 \\
1,7 \\
2,9 \\
0,7 \\
4,1 \\
0,9 \\
6,3 \\
5,7 \\
1,0 \\
-0,2\end{array}$ \\
\hline \multicolumn{5}{|c|}{ (chiffres nominaux) } \\
\hline $\begin{array}{l}\text { Déficit public en } \% \text { du PIB } \\
\text { Déficit public (en milliards } € \text { ) } \\
\text { Actifs (en millions) } \\
\text { Chômeurs indemnisés (en millions) }\end{array}$ & $\begin{array}{r}0,0 \\
-1,0 \\
40,279 \\
3,268\end{array}$ & $\begin{array}{r}-3,3 \\
-79,3 \\
40,265 \\
3,423\end{array}$ & $\begin{array}{r}-4,9 \\
-121,3 \\
40,233 \\
3,382\end{array}$ & $\begin{array}{r}-4,2 \\
-105,0 \\
40,249 \\
3,313\end{array}$ \\
\hline
\end{tabular}

Source des données : Gemeinschaftsdiagnose Frühjahr 2010 (15-04-2010). Les prévisions prennent pour hypothèse notamment un prix moyen du baril de partiellement comparables à celles des rapports de printemps et d'automne 2009 (voir REA 91/09 et 94/09), les Instituts ayant révisé leurs calculs depuis.

Si la tendance à la reprise s'affirme, ses manifestations n'en sont pas moins irrégulières : après deux trimestres d'expansion, les activités ont stagné durant l'hiver. Mais depuis le début de l'année, les carnets de commande de l'industrie se remplissent rapidement ; les exportations, en hausse de $4,5 \%$ au second semestre 2009 , ont même enregistré en mars 2010 un bond de 10,7 \% par rapport à février (+23,3\% par rapport à mars 2009 ; Destatis). Au premier trimestre 2010, le PIB a crû de 0,2 \% (Destatis). Le commerce extérieur a été dynamisé par le retour de la croissance dans les économies émergentes asiatiques et les pays est-européens (les ventes en Chine ont fait un bond de $+13,8 \%$ au second semestre 2009). La demande des pays de la zone euro a connu une légère hausse, en partie imputable aux programmes de soutien conjoncturel adoptés. Les acteurs économiques se montrent confiants en l'avenir, comme le révèle notamment l'indice ifo. Au total, le principal moteur de l'économie allemande redémarre, et la contribution de l'export à la croissance devrait se situer à 0,9 point de pourcentage en 2010 (à 0,6 en 2011).

Dans ce contexte, les investissements redémarrent eux aussi. Certes, le taux d'utilisation des capacités reste faible, mais les entreprises semblent vouloir combler le retard accumulé pour le renouvellement et la modernisation de leurs équipements. La fin programmée en décembre prochain du régime avantageux des amortissements les incite en outre à anticiper leurs investissements. Les commandes domestiques s'accroissent ainsi depuis le milieu de 2009, tirant la production dans la construction mécanique. Cette tendance devrait gagner en ampleur avec le desserrement du crédit aux entreprises.

La consommation des ménages reste stable ; en 2009, la baisse des revenus liée à la récession a été compensée par les mesures de soutien à la demande d'un montant total de 23 milliards $€$, soit un coup de pouce de 1,5\% au pouvoir d'achat. Le revenu disponible des ménages stagnera cette année en termes réels, avant d'augmenter légèrement l'an prochain sous l'effet du regain de rentabilité des placements, la progression des salaires restant modérée après la hausse des coûts salariaux unitaires et la baisse de la productivité horaire liées à la récession. L'inflation devrait être de l'ordre de $1 \%$. Au total, la con-

Franche reprise des exportations

Hausse des investissements

Fin de la morosité de la consommation 
Un taux de chômage de $8,1 \%$ seulement cette année

Pour réduire les risques, priorité à la consolidation budgétaire sommation privée stagnera en 2010 mais contribuera à hauteur de 0,5 point de pourcentage à la croissance en 2011.

En 2009, le nombre des chômeurs ne s'est accru que de 185000 : confrontées à une pénurie chronique de main-d'œuvre qualifiée, les entreprises ont préféré " thésauriser leurs salariés ॥ (Instituts) en épuisant toutes les possibilités de flexibilisation et en recourant au chômage partiel. S'y ajoutent des tendances structurelles dont les Instituts, de leur propre aveu, avaient sous-estimé les effets : la baisse (-93 000) de l'emploi régulier a été compensée par la hausse des petits boulots $(+70000)$; alors que, dans la première catégorie, le nombre des temps plein chutait $(-360000)$, celui des temps partiel augmentait (+270 000). C'est dans l'industrie qu'ont été détruits le plus d'emplois soumis à cotisations sociales et à temps plein ; et c'est dans les services qu'ont été créés le plus d'emplois à temps partiel. Au total, le taux de chômage devrait s'établir à $8,1 \%$ cette année et à 7,9\% l'an prochain (données administratives). C'est nettement moins que dans la zone euro où la Commission prévoit plus de $10 \%$.

Si l'économie allemande commence à sortir de la récession, portée par une demande mondiale redynamisée, mais aussi par les programmes conjoncturels domestiques et étrangers, l'évolution de sa croissance reste soumise à un certain nombre de risques. Le système bancaire allemand n'est pas encore totalement stabilisé ; la BCE devra forcément revenir un jour ou l'autre à une politique moins expansive. Le principal risque cependant reste la délicate définition d'une stratégie de sortie de crise, européenne et nationale. Les programmes conjoncturels allemands expirent à la fin de cette année ; au total, dépenses et baisse de l'encours fiscal confondus, la récession aura creusé un trou de 90 milliards $€$ dans le budget. La consolidation des finances publiques est maintenant la priorité absolue, pour respecter le Pacte de Stabilité ou les nouvelles dispositions constitutionnelles, mais aussi et avant tout pour préserver la crédibilité du gouvernement allemand auprès des citoyens, des agents économiques et des marchés financiers.

Le gouvernement fédéral a prévu de les consolider dès 2011 - à un moment où la reprise devrait être suffisamment confirmée pour que la « rigueur » n'inhibe pas trop l'activité. Le projet de budget sera présenté en juin. Les Instituts estiment que ce planning est très juste, puisqu'il signifie que le Bund devra réduire son déficit structurel de 0,4 point de pourcentage par an d'ici la fin 2015. Et ils pressent le gouvernement Merkel de présenter le détail des mesures envisagées pour préserver la confiance. Ils lui conseillent vivement une politique de consolidation " qualitative » : strict plafonnement des dépenses publiques (suppression des subventions et niches fiscales, modération salariale dans la fonction publique et gains d'efficience dans le système de santé) accompagné d'un redéploiement des dépenses vers l'investissement dans les infrastructures et le capital humain. La coalition fédérale, inhibée dans son action par l'épée de Damoclès des élections de Rhénanie du NordWestphalie du 9 mai, vient de mettre fin à un contentieux entre Union et FDP : il n'y aura " pas de baisse d'impôts en 2011 et 2012 " a déclaré la chancelière à la presse au lendemain des élections. Les économistes n'avaient cessé de critiquer cette revendication du FDP à laquelle ils reprochaient son profond irréalisme. Et dès le 11 mai, les premières propositions d'économies budgétaires formulées par les économistes et divers hommes politiques (dont Roland Koch, ministre-pésident CDU de Hesse) commençaient à circuler.

UN POLITIQUE DE STRICTE RIGUEUR « peut sembler ambitieuse, mais toute autre solution serait pire ", concluent les Instituts dont l'analyse de la conjoncture et de la politique allemandes s'insère dans le contexte européen. Dans la situation actuelle, plusieurs questions se posent. Comment réduire les divergences entre les balances commerciales des Etats membres ? Certainement pas en augmentant les salaires dans les pays excédentaires, car le rôle de la politique salariale "n'est pas de contribuer à résoudre les problèmes de compétitivité des Etats partenaires ». A chacun de remédier à ses propres problèmes, résument les Instituts. Les spéculations attisées par le déficit public grec ont révélé crûment les déficits de la gouvernance de l'UE et de la zone euro. L'effort politique prioritaire est désormais de trouver un " cadre réglementaire garantissant que les objectifs de croissance à long terme ne puissent être mis en danger par la poursuite d'objectifs à court terme » (Instituts). Le projet de loi " pour la stabilisation de la zone euro », adopté par le gouvernement fédéral le 11 mai et dans lequel l'Allemagne s'engage à garantir un montant de 123 milliards $€$ de crédits dans le cadre du plan européen du même nom, n'est qu'un élément adopté dans l'urgence pour rétablir la confiance, appelant une réflexion de fond sur le fonctionnement durable de l'UE et de la zone euro. Dans un éditorial au vitriol dans l'hebdomadaire Die Zeit (12-05), l'ex-chancelier Helmut Schmidt (SPD) rappelle le président Sarkozy et la chancelière Merkel à leurs obligations de respect des règles que leurs pays ont définies pour l'UE et l'euro. Wolfgang Schäuble, ministre fédéral des Finances (CDU), rappelait de son côté (Handelsblatt, 06-05) : l'évolution actuelle dans la zone euro " nous montre combien il est important d'avoir des finances publiques saines, et combien nous avons besoin de l'Allemagne comme havre de stabilité pour l'euro ".

Isabelle Bourgeois (12-05-2010) 